\title{
Optical coherence tomography assessment of vessel wall degradation in aneurysmatic thoracic aortas
}

\author{
Eusebio Real ${ }^{\mathrm{a}}$, Alma Eguizabal ${ }^{\mathrm{a}}$, Alejandro Pontón ${ }^{\mathrm{b}}, \mathrm{J}_{\text {. Fernando Val-Bernal }}{ }^{\mathrm{c}}$, Marta Mayorga ${ }^{\mathrm{c}}$, \\ José M. Revuelta ${ }^{\mathrm{b}}$, José M. López-Higuera ${ }^{\mathrm{a}}$, Olga M. Conde $\mathrm{a}^{\mathrm{a}^{*}}$ \\ ${ }^{a}$ Photonics Engineering Group, Dep. TEISA, University of Cantabria, Avda. Los Castros S/N, 39005 \\ Santander, Spain; ${ }^{\mathrm{b}}$ Cardiovascular Surgery Department, Marqués de Valdecilla University Hospital, \\ Medical Faculty, University of Cantabria and IFIMAV, Santander, Spain; ${ }^{\mathrm{C} A n a t o m i c a l ~ P a t h o l o g y ~}$ \\ Department, Marqués de Valdecilla University Hospital, Medical Faculty, University of Cantabria \\ and IFIMAV, Santander, Spain
}

\begin{abstract}
Optical coherence tomographic images of ascending thoracic human aortas from aneurysms exhibit disorders on the smooth muscle cell structure of the media layer of the aortic vessel as well as elastin degradation. Ex-vivo measurements of human samples provide results that correlate with pathologist diagnosis in aneurysmatic and control aortas. The observed disorders are studied as possible hallmarks for aneurysm diagnosis. To this end, the backscattering profile along the vessel thickness has been evaluated by fitting its decay against two different models, a third order polynomial fitting and an exponential fitting. The discontinuities present on the vessel wall on aneurysmatic aortas are slightly better identified with the exponential approach. Aneurysmatic aortic walls present uneven reflectivity decay when compared with healthy vessels. The fitting error has revealed as the most favorable indicator for aneurysm diagnosis as it provides a measure of how uniform is the decay along the vessel thickness.
\end{abstract}

Keywords: OCT, aneurysm diagnosis, ascending thoracic aorta, vessel wall

\section{INTRODUCTION}

An aneurysm is a pathological situation produced by the enlargement of the aorta diameter [1]. If the diameter of the aorta wall exceeds a specific threshold, a risk of vessel rupture appears due to the weakening of the aortic wall. The risk of rupture for a thoracic aneurysm over $5 \mathrm{~cm}$ is $20 \%$, over $6 \mathrm{~cm}$ nearly $40 \%$ and over $7 \mathrm{~cm}$ around $50 \%$. The rupture of an aneurysm carries a risk of death up to $90 \%$ [2]. Under these situations, conventional imaging modalities such as ultrasound and computed tomography are applied to monitor the progressive diameter enlargement. The cardiovascular treatment for an aneurysmatic aorta is based on surgical interventions where a endovascular stent-graft is placed inside a diseased vessel [3]. It would be desirable to know in advance the area where the aortic wall recovers its mechanical characteristics to properly locate the endovascular prosthesis. Sometimes, the risk of aneurysm is not announced with a preliminary aortic diameter enlargement. This is the case of some congenital syndromes as bicuspid aortic valve (BAV) and the Ehlers-Danlos or Marfan diseases. If a tool for vessel wall degradation would be available, specific treatments could be applied in cases where the aorta diameter does not show evidence of the risk of rupture. Degradation of the aortic wall connective tissue has been shown to be a hallmark of aneurysm formation. Collagen and elastin are the two major connective tissues found in the aorta wall. Histologically, elastin fragmentation and degeneration are observed in the aneurysmatic wall [4]. Elastin, the scaffold of the medial lamellae, is found in a reduced concentration as well as in a fragmented organization within tissues of diseased aorta [5].

In this scenario, Optical Coherence Tomography (OCT) reveals as a natural candidate for imaging structures under the surface. In this case, the interest is not focused on how the arterial lumen is occluded, i.e. in case of atherosclerosis, but in what happens under the vessel surface [6]. The disorders in the elastin and smooth muscle cells structure produce a directional anisotropy of the optical properties of the media layer of the aorta that will induce attenuations of the OCT signal. This paper proposes the "ex-vivo" imaging of the wall of ascending thoracic human aorta from aneurysms to find evidences of tissue disorders in its structure that become contrasted with the histopathological gold standard.

Optical Coherence Tomography and Coherence Techniques VI, edited by Brett E. Bouma, Rainer A. Leitgeb, Proc. of OSA Biomedical Optics-SPIE Vol. 8802, 88020G - (C) OSA-2013 SPIE

CCC code: $1605-7422 / 13 / \$ 18 \cdot$ doi: $10.1117 / 12.2032592$ 


\section{MATERIALS AND METHODS}

\subsection{Aorta samples}

In this study, the cardiovascular surgeons provide human aneurysmatic aorta samples collected during cardiovascular surgery (Bentall, Wheat) of aorta. Fresh aorta specimens where maintained into a phosphate saline buffer (PBS). Samples were refrigerated until the OCT measurement was performed between 3-4 hours after the intervention. Each aorta sample was divided into different regions of interest (ROI) of average size $2.5 \times 10 \mathrm{~mm}^{2}$. Different orientations were considered for each ROI: along and across the direction of blood flow. Fiducial markers with India ink delimit the area of each ROI to facilitate co-registration with the histopathological examination. During the image acquisition procedure tissue was continuously re-hydrated with PBS. After the OCT, examined tissue was placed in $10 \%$ buffered formalin solution for the histopathological analysis. Four different histological stains were analyzed: Hematoxylin and Eosin (H\&E), Verhoeff's Van Gieson (EVG) to check the atrophy of elastic tissue and the thinning and loss of elastic fibers, Alcian Blue (AB) at $\mathrm{pH} 2.5$ to determine acidic polysaccharides and some types of mucopolysaccharides and Alphasmooth muscle actin ( $\alpha$-SMA) used as a marker of vascular smooth muscle cells (SMC). Healthy ascending thoracic aortas come from donors of heart transplantations and they act as control tissue. In this study 21 ROIs from 8 patients (6 males, 2 females, average age 66 years) have been analyzed: 17 ROIs are extracted from ascending thoracic aorta with aneurysm (average diameter $55 \mathrm{~mm}$; max. $70 \mathrm{~mm}$; min. $46 \mathrm{~mm}$ ) and 4 from controls. All samples were diagnosed by experienced pathologists through the tests EVG, $\mathrm{AB}$ and $\alpha$-SMA.

\subsection{Swept-source optical coherence tomography system}

The OCT system used (Thorlabs, OCS1300SS) is based on a swept source with a center wavelength of $1325 \mathrm{~nm}$ and a spectral bandwidth (FWHM) of $100 \mathrm{~nm}$. The system provides an axial resolution of $12 \mu \mathrm{m}$ (in air) and $25 \mu \mathrm{m}$ transverse resolution, with a maximum imaging depth of $3 \mathrm{~mm}$ (in air) and $10 \mathrm{~mm}$ width. The sampling resolution for each B-scan image is 512 (axial) x 1024 (transverse) pixels and the sensitivity is 100dB. Images (B-scans) were acquired focusing the probe on the surface of the aorta sample (intima layer) and applying a temporal average of four acquisitions per image. Series of 1024 B-scans were obtained for each ROI to guarantee the match with the histology image. Figure 1 shows how a ROI is imaged from an aorta sample. Different ROIs (along and across blood flow) where delimited on each aorta sample (left). Once a ROI is defined (center) a sweep is performed in the arrow direction to obtain a reflectivity profile (right) along the whole ROI.

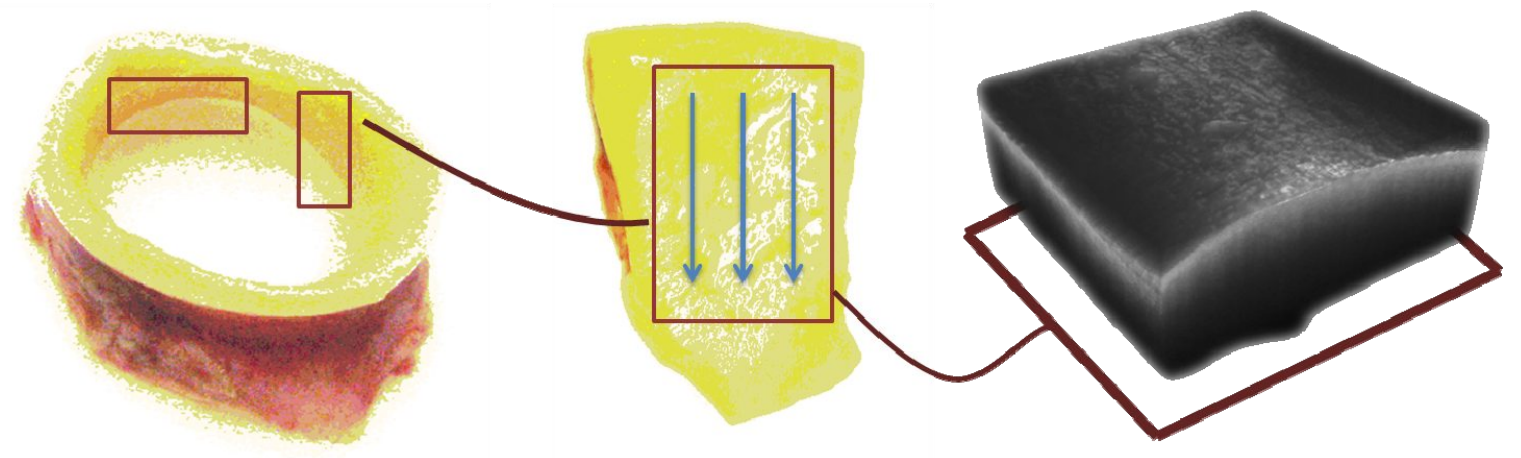

Figure 1. OCT measurement of an aortic vessel: (left) fresh aorta specimen with indication of ROIs; (center) ROI defined on the intima layer of the aortic wall; (right) OCT measurements over the defined ROI.

\subsection{OCT processing}

Arterial walls are layered structures, where each layer has its own functions and particular properties. Hence, it is important to identify layers and align them in order to compare and correlate its properties along the whole wall. OCT images are affected by noise, imaging artifacts and the presence of saline buffer over the sample. In order to automatize the surface and layer recognition, all these effects must be removed: 
- Noise reduction: noise has been minimized considering only the blue component of the RGB false-color image of the OCT measurement. Due to the color representation of the OCT signal, the blue component has little presence in each pixel composition, limited to areas of high intensity.

- Artifacts elimination: sometimes light becomes mostly reflected by the surface not providing any information about the tissue depth and distorting the detection of the air-sample interface. These reflections spoil the whole line of pixels in depth. To avoid this, artifacts should be firstly identified and afterwards removed [7] or corrected. Artifacts are identified as pixels with an intensity level higher than a threshold based on the statistics of a region in the image known to be air [7]. Reconstruction of the artifacts is proposed interpolating the values of adjacent pixels.

- Saline detection: as tissue samples were conserved in saline, sometimes small pools of PBS appear over the vessel surface. The interface air-saline could be confused with the beginning of tissue. To avoid this effect, it has been established a threshold of intensity saturation, to distinguish between saline (higher intensity than air but less than tissue) and real tissue.

Once the blue component of the raw image has been corrected from reflection artifacts, a border recognition method is applied comparing, for every A-scan, the intensity level with a threshold in order to detect the transition from the region of air to the sample. The interface line obtained is then smoothed applying an average related with its neighborhood. Once the border has been revealed in the blue component, the original image can be cropped, removing the region of air. Figure 2 shows an OCT measurement of an aneurysmatic aortic wall. On the left image, the OCT image is presented with a superimposed pink line that identifies the air-sample interface. The right image is the result of automatically crop the part of the OCT image that corresponds with air.
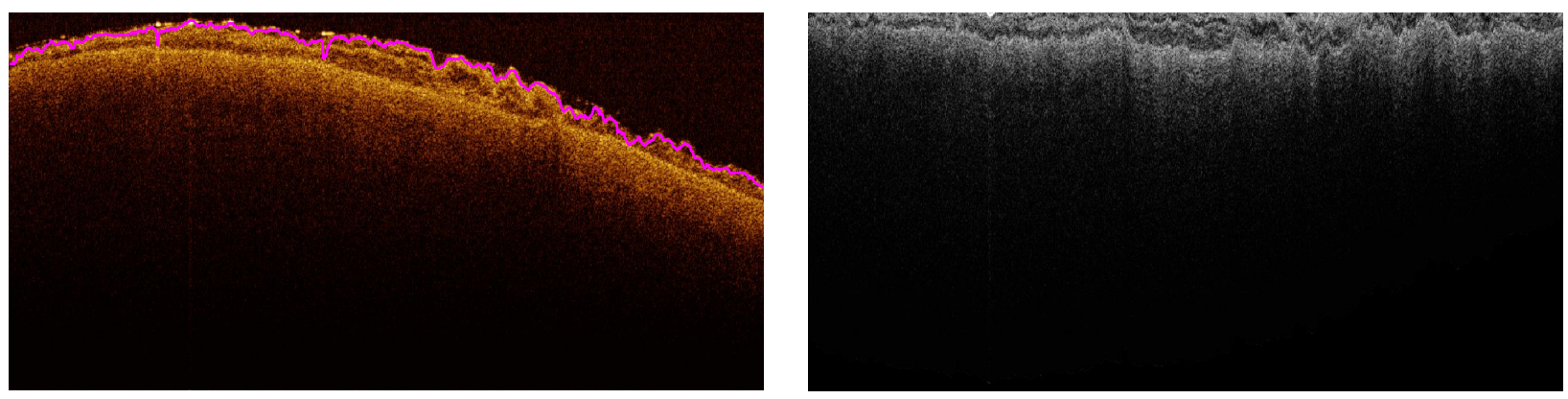

Figure 2. Identification of the air-sample interface on a OCT measurement: (left) original OCT image with the pink line representing the air-sample interface; (right) OCT image cropped with the air part removed.

\subsection{Backscattering model}

The structure of an aortic wall is composed by three different layers: the intima layer composed primarily by endothelium, the media layer composed of smooth muscle cells and elastic fibers and the adventitia with mostly collagen fibers. The maximum imaging depth of the OCT system only allows to obtain information about the first two layers (intima and media) but this is enough for characterization purposes. As stated, the media layer exhibits the most interesting histological features for the evaluation of the risk of aneurysm. After the preliminary air-sample interface detection, the border between the intima and the media layers should be identified to remove the intima contribution to the OCT measurement. The assessment of pathologists indicates that the medium thickness of the healthy intima is 52 micron, whereas the thickness of aneurysmatic intima is around 62 microns. Taking an approximate refraction index for aorta wall of 1.35 [8], this implies a thickness between 12 to 15 pixels on each OCT images from the air-sample interface. A safety thickness of 20 pixels has been selected for the segmentation of the intima.

As known, on an OCT measurement, each B-scan image comes from concatenated A-scan measurements, each one representing the backscattering evolution of tissue along the depth. Each A-scan, or reflectivity profile, can be analyzed individually. To this end, the amplitude of the OCT signal reflectivity decay along the depth of the media layer of the aortic wall is fitted against different laws. It is hypothesized that as the wall structure of healthy and aneurysmatic vessel differs, the same does the reflectivity. Therefore, the changes in the decay law from both clinical cases could become diagnosis hallmarks for the risk of aneurysm. 
After isolating the media layer, the backscattering signal is fitted against different models, two reflectance decays are considered: an exponential law and a polynomial curve. The exponential decay has been chosen to be consistent with the expected evolution, according to the Beer-Lambert law, of the optical backscattering signal I(z) along the penetration depth. This modeling is shown on Eq. 1 where $z$ is the optical depth, $\alpha$ collects the deviation on attenuation and $A$ would be proportional to backscattering amplitude. In the polynomial approach, Eq. 2, there is no such a direct relation with the optical properties of tissue, but interesting backscattering changes can be also collected. In the polynomial approach, $a$ is the cubic, $b$ the quadratic and $c$ the linear term respectively.

$$
\begin{gathered}
I(z)=A e^{\alpha z}+C \\
I(z)=a z^{3}+b z^{2}+c z+d
\end{gathered}
$$

In both approaches, exponential and polynomial, independent terms $(C$ and $d)$ have been added to compensate for background differences among samples. This way, results of the fitting parameters of control and aneurysmatic specimens can be compared, concluding rather the exponential or the polynomial models coefficients are relevant for automatic characterizations and classification of the risk of aneurysm.

\section{RESULTS AND DISCUSSION}

For all the investigated samples, a total of 21,504 B-scan images have been analyzed. Aortas from aneurysm and healthy patients are shown in Fig. 3 and 4. In the case of aneurysmatic aortas, the elastin perturbation in EVG (Fig.3b) correlates with acid mucopolysaccharides deposit (Fig.3c) showing cystic medial degeneration with extensive destruction of elastic fibers and focal loss of smooth muscle cells. OCT (Fig.3a) and PS-OCT (Fig.3d) images also reveal these perturbations confirming this analysis feasible for aortic wall characterization.
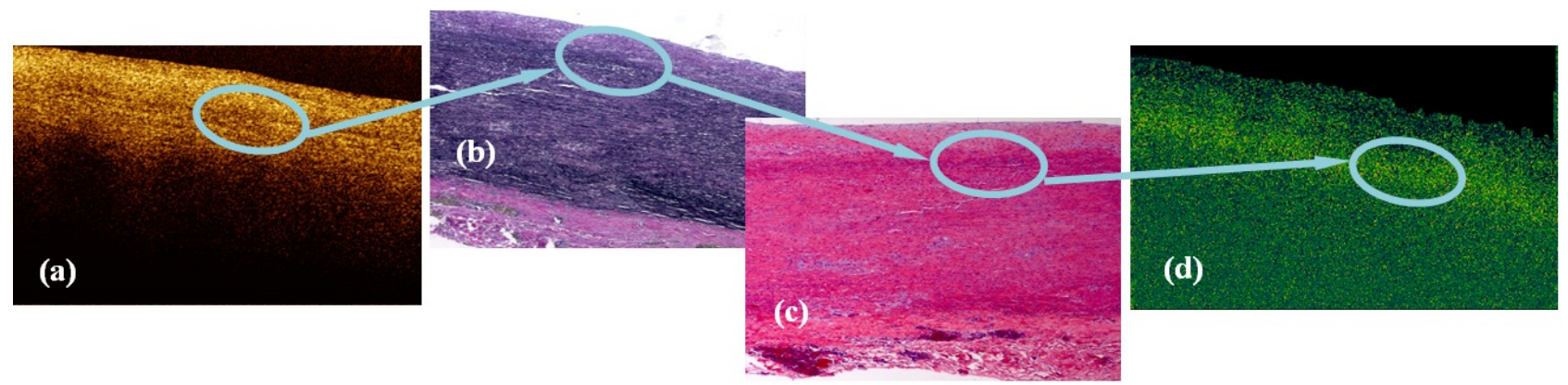

Figure 3. Aorta wall from an aneurysm: (a) OCT intensity; (b) EVG stain; (c) AB stain; (d) PS-OCT.

In the case of healthy walls from controls with normal ascending thoracic aortas, histology does not exhibit significant alterations (Fig.4b) as well as the OCT (Fig.4a) and PS-OCT (Fig.4c) images. A quasi-uniform decay of the A-scan profile could be expected in this case.
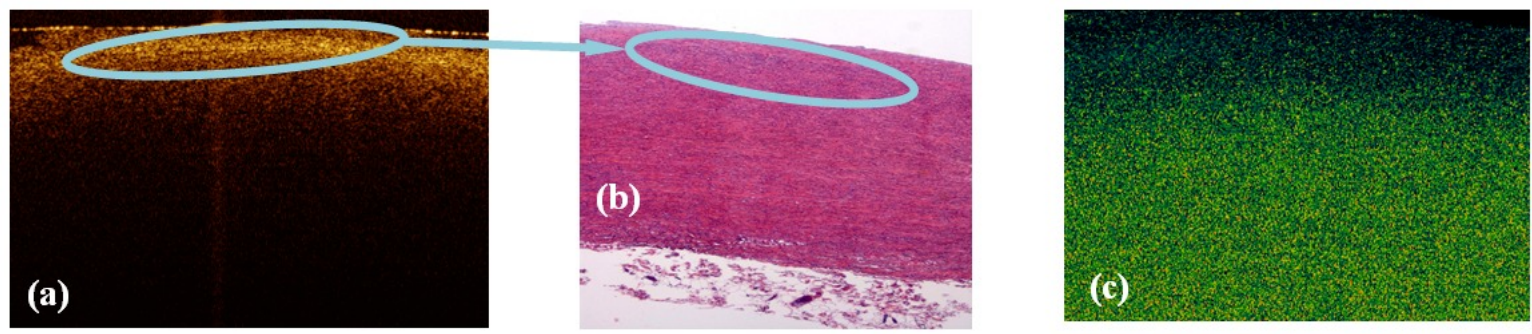

Figure 4. Healthy aorta wall from a control: (a) OCT intensity; (b) AB stain; (c) PS-OCT. 
Figure 5 represents how the fitting approach behaves for each one of the models presented by Eq.1 and Eq.2 and for different diagnostic categories: healthy and aneurysmatic aortas. The left image of Figure 5 represents one of the A-scans of a healthy aortic wall. Although some peaks are present on the reflectivity profile, a more uniform decay can be observed. When the exponential approach (dashed red) is applied, a Pearson's correlation coefficient of 0.99 is achieved. This reflects that a better match between the model and the measurement is achieved. In this case of healthy aortas, the polynomial law (dotted blue) presents a slightly worst behavior. However, when the media layer of an aneurysmatic wall is analyzed, right graph of Figure 5, its A-scan (continuous black) presents a more disordered decay with an increased presence of peaks in the reflectivity profile that account for the stated disorders in the tissue structure. In this case, both models, exponential and polynomial, are unable to match with accuracy the backscattering profile.
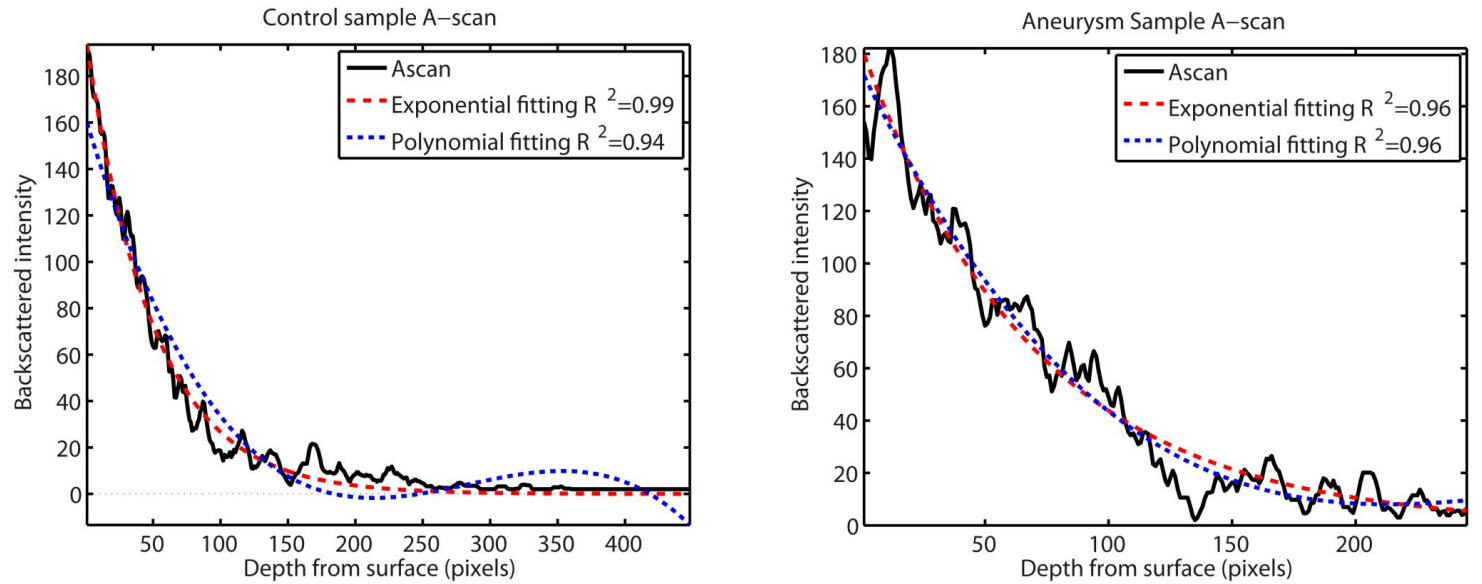

Figure 5. A-scan section of the media layer of the aorta wall: healthy aorta (left) and aorta from an aneurysm (right).

In order to check if a compact indicator for aneurysm diagnosis could be found, Figures 6 and 7 present the boxplots diagrams corresponding with the diagnosis ability of each one of the parameters of the fitting models. Figure 6 represents the boxplots of the polynomial coefficients and Figure 7 does the same for the exponential parameters. In general, a wider statistical distribution is always found in the aneurysmatic category, whereas the healthy samples present less dispersion. This reflects again the heterogeneity that can be found in pathological aortas. In the case of the polynomial fitting, only the quadratic and the linear terms are presented, being the quadratic parameter the one where the medians of categories (red lines) appear to be slightly more separated. However, the fitting error measured as a function of the Pearson's correlation coefficient seems to provide the better diagnosis ability in accordance with the analysis performed when the A-scan profile where discussed.
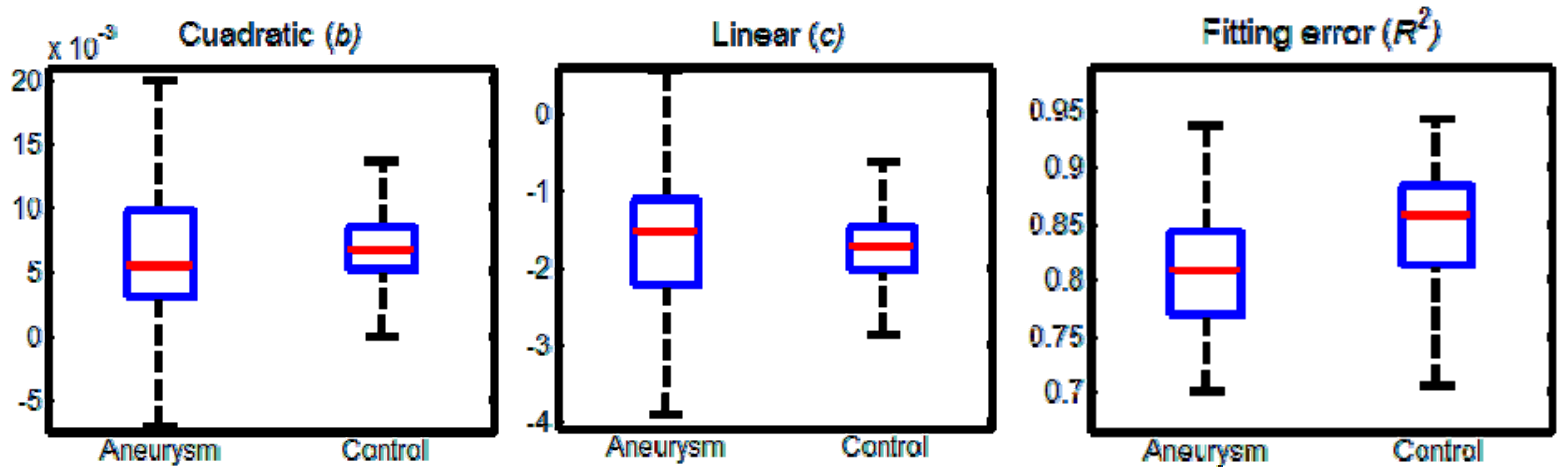

Figure 6. Boxplots of the diagnosis ability of the parameters of the polynomial fitting: (left) quadratic term, $b$; (center) linear term, $c$; (right) fitting error as a function of the Pearson's correlation coefficient. 
In the case of the exponential fitting, Figure 7, almost the same conclusion can be obtained. The more discriminant ability is again obtained by the fitting error where slightly more clustered categories could be found.
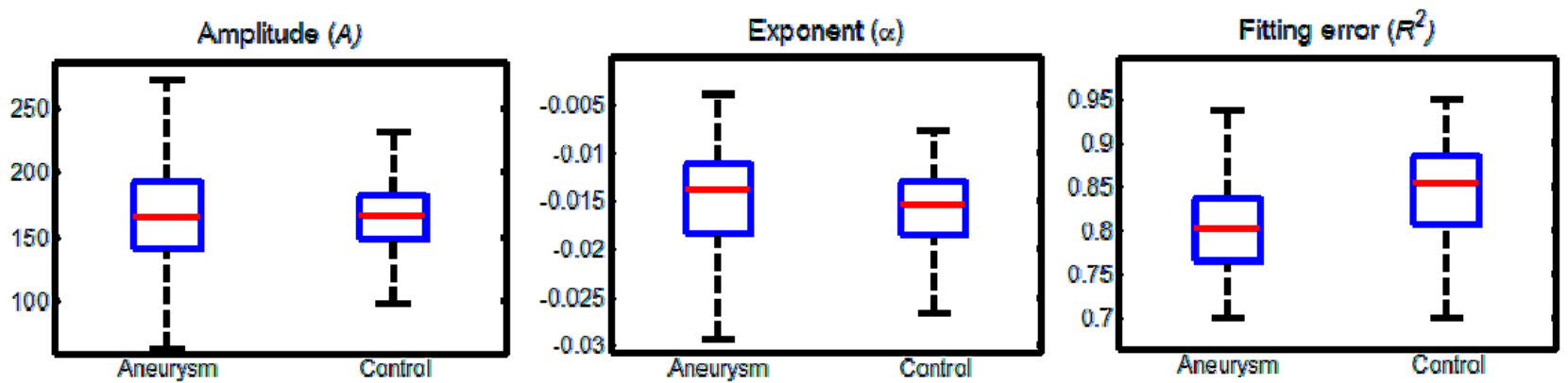

Figure 7. Boxplots of the diagnosis ability of the parameters of the exponential fitting: (left) backscattering amplitude, $A$; (center) attenuation, $\alpha$; (right) fitting error as a function of the Pearson's correlation coefficient.

Finally, and to validate the diagnosis performance of each parameter, the Receiver Operating Characteristic (ROC) has been computed [9]. In this case, the area under the ROC curve (AUC) will be considered as a figure of merit to evaluate the accuracy of a feature for classification. If the area under the ROC curve of a specific parameter results bigger than 0.7 , i.e. $70 \%$, it will be considered appropriate to diagnose aneurysmatic conditions. In the case of the exponential fitting the AUC achieved by the fitting error parameter $R^{2}$ is 0.72 , whereas in the case of polynomial fitting is 0.70 .
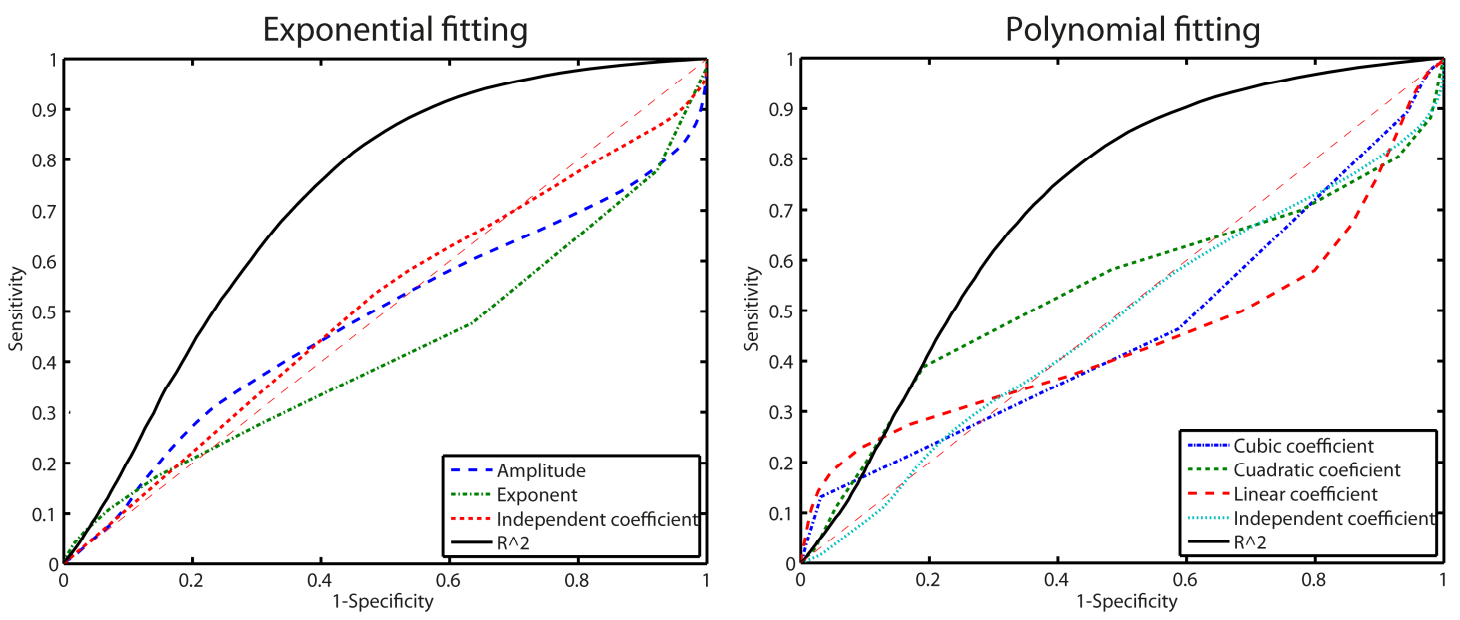

Figure 8. ROC curves for each approach: (left) exponential fitting; (right) polynomial fitting.

\section{CONCLUSIONS}

The morphological variations in arterial tissues generate changes in the optical properties that can be addressed by the OCT technique. The degradation of the elastin content and the disorders of the smooth muscle cells structure in the media layer of the aortic wall under aneurysm conditions appear as intensity signal perturbations in the OCT measurements. Ex-vivo measurements of human samples of ascending thoracic aortas have been performed. Two diagnostic categories have been analyzed: aortas from aneurysms and from healthy patients considered as controls. After the OCT measurement, samples were sent to the pathologist. The backscattering profiles of the OCT measurements have been modeled following two laws: exponential and polynomial. Prior to the fitting process, the OCT image must be conditioned in terms of noise and artifacts reduction and air-sample interface detection. The intima layer contribution has also been removed applying the pathologist assessment. The fitting error of the model approximation has been found as 
the best indicator for aneurysm diagnosis. The diagnosis ability of the fitting error is slightly better in the case of the exponential approach where an AUC of 0.72 has been achieved. This result provides a proof of principle for this optical imaging modality and encourages further investigation towards the automatic detection of these perturbations.

\section{ACKNOWLEDGEMENTS}

This work has been supported by the Spanish Government through CYCIT projects DA2TOI (FIS2010-19860), TFS (TEC2010-20224-C02-02) and the Alma’s Eguizabal PhD Grant (FPU12/04130).

\section{REFERENCES}

[1] Johnston, K.W., Rutherford, R.B., Tilson, M.D., Shah,D.M., Hollier,L. and Stanley, J.C., "Suggested standards for reporting on arterial aneurysms. Subcommittee on Reporting Standards for Arterial Aneurysms, Ad Hoc Committee on Reporting Standards, Society for Vascular Surgery and North American Chapter, International Society for Cardiovascular Surgery," Journal of Vascular Surgery 13, 452-458 (1991).

[2] Clouse, W.D., Hallett, J.W., Schaff, H.V., Gayari, M.M., Ilstrup, D.M., and Melton, L.J. 3rd., "Improved prognosis of thoracic aortic aneurysms: a population-based study,” JAMA 280, 1926-1929 (1998).

[3] Hiratzka, L.F., Bakris, G.L., Beckman, J.A., et al., "Guidelines for the diagnosis and management of patients with thoracic aortic disease: executive summary," Journal of the American College of Cardiology 55, 15091544 (2010).

[4] Rizzo,R.J., McCarthy, W.J., Dixit,S.N., Lilly,M.P., Shively,V.P., Flinn W.R., et al., "Collagen types and matrix protein content in human abdominal aortic aneurysms" Journal of Vascular Surgery 10, 365-373 (1989).

[5] Mecham,R.P., Broekelmann,T., Davis,E.C., Gibson,M.A., and Brown-Augsburger,P., "Elastic fibre assembly: macromolecular interactions" in Ciba Found Symp. 192, 172-181 (1995).

[6] Popescu, D.P., Flueraru,C., Mao,Y., Chang,S., Sowa, M.G., "Signal attenuation and box-counting fractal analysis of optical coherence tomography images of arterial tissue," Biomedical Optics Express 1, 268-277 (2010).

[7] Garcia-Allende,P.B., Amygdalos,I., Dhanapala,H., Goldin,R.D., Hanna, G.B., and Elson,D.S., "Morphological analysis of optical coherence tomography images for automated classification of gastrointestinal tissues," Biomedical Optics Express 2, 2821-2836 (2011).

[8] van der Meer, F.J., Faber, D.J., Çilesiz,I., van Gemert, M.J.C. and van Leeuwen,T.G., "Temperature-dependent optical properties of individual vascular wall components measured by optical coherence tomography," J. Biomed. Opt. 11, 041120 (2006).

[9] Fawcett,T. “An introduction to ROC analysis," Pattern Recogn. Lett. 27, 861-874 (2006). 